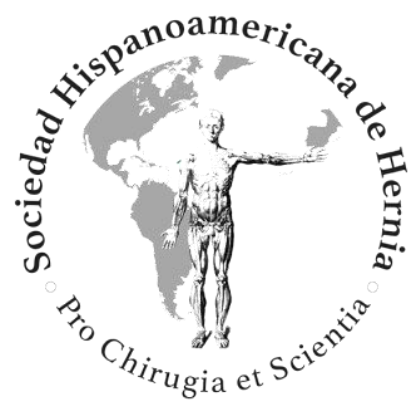

Utilidad del drenaje en las eventroplastias con malla

Utility of drainage in incisional hernia repair with mesh 


\section{Utilidad del drenaje en las eventroplastias con malla}

Utility of drainage in incisional hernia repair with mesh

Jimena Belén Torres Tejerizo ${ }^{1}$, Jorge Daniel Bartoli ${ }^{1}$, Eduardo Dreizzen ${ }^{2}$

${ }^{1}$ Hospital Italiano La Plata. La Plata (Argentina). ${ }^{2}$ Facultad de Ciencias Médicas. La Plata (Argentina)

Autor para correspondencia: Jimena Belén Torres Tejerizo. Hospital Italiano. Av. 51, B1900 La Plata (Argentina)

Correo electrónico: jimenita_bqc@hotmail.com

Recibido: 04-12-2019

Aceptado: 13-12-2019

El original ha sido presentado previamente como trabajo de tesis doctoral de Jimena Belén Torres Tejerizo en la Facultad de Ciencias Médicas de La Plata. Universidad Nacional de La Plata (Argentina). 20 de marzo de 2019.

\section{RESUMEN}

Introducción: La reparación de las eventraciones presenta una alta tasa de complicaciones, entre las que el seroma y la infección del sitio quirúrgico están entre las más frecuentes. Los drenajes se usan durante la reparación de una eventración para facilitar la salida del líquido y prevenir la aparición de complicaciones. No existe consenso sobre su utilidad.

Objetivo: Nuestro objetivo fue determinar si la utilización de drenajes en las eventroplastias con malla influye en la aparición de complicaciones.

Métodos: Estudio observacional de cohortes, prospectivo y comparativo de dos grupos de pacientes operados de eventración, unos con colocación de drenaje y otros, sin ellos. 
Resultados: Incluimos 102 pacientes, 42 en el grupo con drenaje y 60 en el grupo sin drenaje. Encontramos un total de 69 complicaciones. La más frecuente fue la presencia de seroma, seguida de la infección de la herida quirúrgica.

Conclusiones: La aparición de complicaciones en una intervención quirúrgica depende de múltiples factores: la edad, la diabetes y el estado nutricional parecen ser los más significativos. Los pacientes con IMC elevado ( $\geq 30$ ) y eventraciones $\geq 10 \mathrm{~cm}$ desarrollaron con mayor frecuencia complicaciones de la herida. No encontramos que la colocación de un drenaje en las eventroplastias con malla influya en la aparición de complicaciones durante el posoperatorio.

\section{ABSTRACT}

Introduction: Repair of incisional hernia presents a high rate of complications, seroma and surgical site infection are among the most frequent. Drains are often placed during the repair of an incisional hernia to facilitate leakage of the liquid and prevent the onset of complications. There is no consensus on its usefulness.

Objective: Our objective was to find out whether the use of drainage in incisional hernia repair with mesh influences the appearance of complications.

Methods: We carry out an observational cohort study, prospectively compared two groups of operated patients, some of them with drainage placement and some others without them.

Results: We included 102 patients, 42 in the drainage group and 60 in the non-drainage group. We found in total 69 complications, the most frequent complication was the presence of seroma, followed by the infection of the surgical wound.

Conclusions: The onset of complications in a surgical intervention depends on multiple factors; age, diabetes and nutritional status seem to be the most significant ones. Patients with high BMI ( $\geq 30$ ) and incisional hernias $\geq 10 \mathrm{~cm}$ developed more frequently wound complications. We do not find that the placement of drainage in the 
repair of incisional hernia with mesh influences the appearance of complications during the postoperative period.

Palabras clave: Eventroplastia, drenaje, malla, complicaciones.

Keywords: Incisional hernia repair, drain, mesh, complications.

\section{INTRODUCCIÓN}

Las hernias incisionales o eventraciones son causadas por un defecto en la pared abdominal después del cierre de una laparotomía. Sin duda, la eventración es la complicación posquirúrgica tardía más frecuente en cirugía visceral. En Estados Unidos se operan cerca de 250000 casos al año, con una tasa de incidencia próxima al $20 \%$, cifra seguramente subestimada. La reparación de una eventración es exclusivamente quirúrgica ${ }^{1}$.

Los drenajes se colocan frecuentemente durante la reparación de una eventración con el objetivo de facilitar la salida del líquido y prevenir la aparición de complicaciones. No existe consenso entre los cirujanos sobre la necesidad de colocar un drenaje en las distintas reparaciones y sus ventajas en la prevención de complicaciones de las heridas, por lo que algunos los utilizan rutinariamente $y$ otros, en raras ocasiones s.3. $^{2,3}$.

Los cirujanos que colocan drenaje se basan en que este procedimiento reduce la acumulación de líquido y de fluidos en las heridas. Algunos sostienen que la formación de un hematoma actuaría como predisponente para la recidiva de la eventración ${ }^{4}$, por lo que el drenaje sería profiláctico. Quienes no lo colocan argumentan que la presencia de un drenaje no altera la evolución de las heridas y que incluso podría actuar como cuerpo extraño y facilitar la vía de ingre $<<$ so de bacterias, lo que incrementa las posibilidades de infección ${ }^{5}$.

No hay evidencia científica que asegure que la presencia de drenaje disminuye las complicaciones de la herida después de la reparación de las hernias incisionales 5 . Ante esta falta de estudios se planteó el 
presente trabajo, que tiene como objetivo analizar si los drenajes inciden en la evolución de las heridas de las eventroplastias.

\section{MATERIALES Y MÉTODOS}

Se realizó un estudio observacional de cohortes, cuyo criterio de selección fue la presencia o no de drenaje. Al inicio del estudio todos los individuos incluidos estaban libres de la enfermedad o evento que quería estudiarse. Se realizó un seguimiento prospectivo registrando la aparición de complicaciones de la herida quirúrgica.

Para conocer el tamaño muestral que garantizara que el objetivo del estudio fuera adecuadamente respondido con la muestra seleccionada, se realizó el cálculo del tamaño muestral para una proporción $^{6,7}$. Definimos la prevalencia de complicaciones posoperatorias en eventroplastias, estimada en $15 \%$ según el consenso del Panel Latinoamericano de Hernias y aceptamos un margen de error $(\alpha)$ del $5 \%$. Calculamos una muestra no menor a 82 pacientes para completar el estudio y obtener resultados estadísticamente significativos.

El presente trabajo se realizó en el Servicio de Cirugía General del Hospital Italiano de La Plata (Argentina), e incorporó a todos los pacientes que ingresaron para cirugía programada por presentar una eventración abdominal. Incluimos en el estudio a todos aquellos pacientes que ingresaron para eventroplastia abdominal con colocación de malla protésica, mayores de 18 años, sin distinción de sexo, en el Hospital Italiano de La Plata, durante el periodo 20142017.

El primer contacto con el paciente se realizó por consultorios externos a cargo de un cirujano del personal, que daba aviso al investigador responsable, encargado de determinar si el participante era apto para ser incluido en el estudio y si estaba de acuerdo con su inclusión. El protocolo de estudio fue revisado y autorizado por el Comité de Ética del Hospital Italiano de La Plata. El día de la cirugía se realizó la firma del consentimiento informado, en el que se explicaba a los pacientes 
en qué consistía la investigación y solo si estaban de acuerdo se los consideraba participantes.

\section{Protocolo quirúrgico}

La intervención se practicó por vía abierta, resecando la cicatriz previa del paciente y procediendo con la apertura del tejido celular subcutáneo con electrobisturí. El aislamiento del saco eventrógeno y su separación de los tejidos adyacentes se efectuó con disección roma y electrocauterio. El saco eventrógeno se abrió para revisar su contenido, asegurando que ningún asa intestinal quedara atrapada al reintroducir el contenido en la cavidad abdominal. En caso de tener suficiente tejido, la línea media se restituía mediante la sutura continua de sus bordes ${ }^{8}$. La reaproximación de los músculos rectos en la línea media es una recomendación del Ventral Hernia Working Group, y tiene su justificación en la restauración de la funcionalidad de la pared abdominal ${ }^{9}$. Se colocó una malla protésica fijada con material irreabsorbible al plano supraponeurótico. Se realizaron puntos a nivel del tejido celular subcutáneo para disminuir el espacio disecado. En los casos en los que el cirujano decidió colocar un drenaje, se utilizó uno aspirativo (drenaje tipo Jackson Pratt de $7 \mathrm{~mm}$ ), localizado a nivel del tejido celular subcutáneo, que se exteriorizó por contraabertura.

La evaluación y el seguimiento ambulatorio se realizaron a los siete días para el primer control y a los 15 días, coincidiendo con la fecha estipulada por el especialista a cargo para extracción de puntos. A su vez, pasados seis meses de posoperatorio se realizó una entrevista telefónica a los pacientes para finalizar así el seguimiento.

\section{Análisis estadístico}

El estudio se llevó a cabo durante tres años. Pudimos realizar un seguimiento mínimo de seis meses. Los datos obtenidos fueron recolectados y analizados comparando los resultados de ambos grupos. Realizamos una evaluación estadística con el objetivo de 
demostrar si la colocación de drenaje influía en la aparición de complicaciones. Las variables cuantitativas se describen mediante el valor de la media \pm desvío estándar (DS) y las variables cualitativas mediante porcentajes. Se utilizó el test de t de student para evaluar diferencias entre las variables cuantitativas y el test de chi cuadrado para analizar las variables cualitativas. Un valor de $p<0,05$ se consideró estadísticamente significativo ${ }^{6,10}$.

\section{RESULTADOS}

Participaron en el estudio 102 individuos. La muestra obtenida se dividió en dos grupos de estudio con base en la colocación de drenaje. El grupo A, formado por 42 pacientes, tuvo un drenaje colocado, mientras que el $\mathrm{B}$, integrado por 60 pacientes, no tuvo drenaje. Con excepción del sexo, las variables antropométricas en ambos grupos no presentaron diferencias estadísticamente significativas (tabla I).

El tamaño de la eventración se constató midiendo el diámetro máximo del anillo. Encontramos los defectos de 3 a $6 \mathrm{~cm}$ como los más frecuentes. La distribución en ambos grupos puede apreciarse en la tabla II. Puede observarse una diferencia estadísticamente significativa respecto al tamaño de la eventración y la colocación de drenaje, lo que podría deberse a no haber realizado la distribución de los pacientes de forma aleatorizada. No fue posible analizar la utilidad del drenaje en los pacientes con eventraciones menores a $3 \mathrm{~cm}$ debido a que a ninguno de estos pacientes se le colocó drenaje.

A los 42 pacientes del grupo A se les colocó un drenaje tipo Jackson Pratt. El tiempo promedio de extracción del drenaje fue de 3.1 días, y no se evidenció ninguna complicación al momento de su retirada. Un $52.9 \%$ (54 pacientes) evolucionó sin complicaciones, en tanto que el 47.1 \% (48 pacientes) tuvo alguna complicación en el posoperatorio inmediato.

En el grupo con drenaje encontramos el mismo número de pacientes con y sin complicaciones (21 pacientes). Por otra parte, en el grupo 
sin drenaje, 27 pacientes desarrollaron complicaciones, mientras que 33 no las tuvieron. No encontramos diferencia estadísticamente significativa en el desarrollo de complicaciones a partir de la colocación de drenaje ( $p=0.619$ ) (fig. 1). En la tabla III se resumen dichos hallazgos.

Durante el posoperatorio, 48 pacientes afrontaron alguna complicación, pero su número total ascendió a 69 debido a que en algunos casos se asociaron varias complicaciones en el mismo paciente. En ambos grupos, el seroma fue la complicación desarrollada con mayor frecuencia, con una incidencia del $42.1 \%$, y en segundo lugar, la infección de la herida, con una incidencia del 10.8 \%. En cuanto a su aparición respecto a la colocación de drenaje, encontramos que los pacientes que evolucionaron con seroma en el grupo con drenaje fueron el $45.2 \%$, mientras que dentro del grupo sin drenaje fueron el $40 \%$. La diferencia entre el desarrollo de seromas y la colocación de drenaje no fue estadísticamente significativa ( $p=$ 0.598) (fig. 2). Vimos que la aparición de seroma se produjo durante el primer mes posoperaorio, por lo que su colocación y retiro en los primeros dias tendría poca utilidad para su prevencion.

La segunda complicación encontrada en frecuencia fue el desarrollo de infecciones de la herida operatoria. En el grupo con drenaje, su aparición se constató en el $16.7 \%$, mientras que en el grupo sin drenaje fue del $6.7 \%$. Sin embargo, esta diferencia no fue estadísticamente significativa ( $p=0.109$ ) (fig. 3 ).

Hay factores que predisponen a la aparición de complicaciones en la cirugía general. Decidimos evaluar en nuestra muestra si estas variables fueron factores de riesgo en la aparición de complicaciones. Evaluamos algunos factores de riesgo como el sexo, la edad, la presencia de diabetes, enfermedades pulmonares crónicas, neoplasias, índice de masa corporal y tamaño de la eventración. Encontramos que la edad $\geq 50$ años, la diabetes, el IMC $\geq 30$ y las eventraciones $\geq 10 \mathrm{~cm}$ fueron factores estadísticamente significativos en la aparición de complicaciones posoperatorias (tabla IV) 


\section{DISCUSIÓN}

Si bien la recolección de datos se realizó de forma prospectiva, la ubicación de los pacientes en ambos grupos de estudio no fue aleatorizada, lo que podría constituir una limitación del estudio. Sin embargo, como se presentó en la tabla I, no encontramos diferencias estadísticamente significativas en las características de los pacientes de ambos grupos. Este estudio muestra que la colocación de drenaje en la reparación abierta con malla de las eventraciones no influye en la aparición de complicaciones posoperatorias.

La mayoría de los autores describe la formación de seroma como la complicación más frecuente (entre un 5-30 \% de los casos), aunque algunas series reportan incidencias de hasta un $78 \%{ }^{11}$. En nuestra serie la incidencia de seroma fue del $42.1 \%$, coincidiendo con la bibliografía consultada. La infección de la herida ocuparía el segundo lugar (3-18\%), y en algunos casos puede requerir la extracción de la malla ${ }^{12,13}$. La incidencia de infección en nuestro trabajo fue del 10.8 $\%$.

Algunos investigadores ${ }^{14}$ han informado de la escasa influencia del drenaje en la aparición de seromas, quienes sostienen que el pico en la formación de líquido en la herida ocurre aproximadamente dos semanas después de la intervención; por lo tanto, su colocación y su retiro temprano tendrían poca utilidad en la prevención del seroma ${ }^{15}$. En la mayoría de los casos reportados, se han utilizado drenajes de succión en un intento de prevenir la formación de seroma y hematoma, aunque un grupo enfatizó que el drenaje aspirativo se eliminó lo más pronto posible para reducir el riesgo de infección ${ }^{16}$. Vrijland presenta resultados sugestivos de que no existe relación entre la colocación del drenaje subcutáneo y la disminución en la formación de seroma ${ }^{17}$.

Una revisión del 2013 de la Cochrane Library identificó solo un ensayo clínico que evaluaba la utilidad de los drenajes en la reparación de la hernia incisional, pero la comparación se realizó sobre la base de dos 
tipos de drenajes y no incluía un grupo control. Dicho estudio concluyó que no había evidencia suficiente para determinar si los drenajes en las heridas de las eventroplastias eran beneficiosos en la evolución ${ }^{5}$. El trabajo de Westphalen, posterior a ese estudio, evaluó a 42 pacientes intervenidos quirúrgicamente por eventraciones y no detectó diferencias en la formación de seroma o infección a partir de la colocación de drenaje ${ }^{14}$, lo que es similar a lo observado en nuestra serie.

Otros autores sostienen que la utilidad que conlleva la colocación de drenaje es la evacuación del líquido que se genera en la herida, propio de la reacción inflamatoria del organismo, para que no actúe como medio de cultivo para posteriores infecciones del sitio quirúrgico ${ }^{18}$. Correa Alfonso realizó una investigación sobre la reparación de hernias incisionales mediante mallas en posición prefascial o supraponeurótica en la que no se reportaron complicaciones inmediatas como seroma o hematoma de la herida quirúrgica, al haberse colocado en todos los casos un drenaje de la zona ${ }^{19}$. En el año 2018, Fan realizó el análisis de la utilidad del drenaje en 78 pacientes y demostró un descenso significativo en la formación y el tamaño del seroma después de la hernioplastia laparoscópica ${ }^{20}$. Nuestra experiencia no mostró beneficios en la disminución de seromas en el posoperatorio al colocar un drenaje (45.2\% frente a 40 $\%)$.

\section{CONCLUSIONES}

La aparición de complicaciones en una intervención quirúrgica depende de múltiples factores. Aquellos propios del paciente, como la edad, la diabetes y el estado nutricional, parecieran ser los más significativos. En nuestra serie, los pacientes con IMC elevado ( $\geq 30$ ) y eventraciones mayores o iguales a $10 \mathrm{~cm}$ desarrollaron con mayor frecuencia complicaciones de la herida. No encontramos evidencia que permita indicar que la colocación de un drenaje en las 
eventroplastias con malla prevenga la aparición de complicaciones durante el posoperatorio.

\section{BIBLIOGRAFÍA}

1. Tatay FC, Egea AM. Eventraciones (1.a ed). Valencia: Asociación Española de Cirujanos; 2012.

2. Ergül Z, Akinci M, Yilmaz KB, et al. Why do we use drains in some inguinal hernia repairs? Chir. 2011;106(6):769-74.

3. Schmidbauer R, Ladurner R, Hallfeldt $K$, et al. Heavy-weight versus low-weight polypropylene meshes for open sublay mesh repair of incisional hernias. Eur J Med Res. 2005;(10):247-53.

4. George $C D$, Ellis $H$. The results of incisional hernia repair: a twelve-year review. Ann R Coll Surg Engl. 1986;68(4):185-7.

5. Gurusamy K, Allen V, Samraj K. Wound drains after incisional hernia repair. Cochrane Database Syst Rev. 2013; (12):CD005570. DOI: 10.1002/14651858.CD005570.pub4

6. Daniel WW. Bioestadística (4.a edición). México: Limusa Wiley; 2013.

7. Center for Surveillance E\& LS (CSELS). Epi InfoTM. Division of Health Informatics \& Surveillance (DHIS) [Internet]. Disponible en: www.cdc.gov/epiinfo/index.html

8. Jensen $K$, Kjaer $M$, Jorgensen L. Abdominal muscle function and incisional hernia: a systematic review. Hernia. 2014;18(4):481-6. DOI: 10.1007/s10029-014-1242-8

9. Brandi C. Tratamiento de los defectos de la pared abdominal. Rev Argent Cir. 2009; Número extraordinario.

10. Straus S, Glasziou P, Richardson WS. Evidence-Based Medicine (3.a edición). Elsevier; 2005. pp. 177:197.

11. Birch DW. Characterizing laparoscopic incisional hernia repair. Can J Surg. 2007;50(3):195-201.

12. González Mayagoitia JC, Cisneros Muñoz H, Martínez Munive A. Guías de práctica clínica para hernias de la pared abdominal. Asoc Mex Hernia. 2015;1-46. 
13. Cassar K, Munro A. Surgical treatment of incisional hernia. $\mathrm{Br}$ J Surg. 2002;89(5):534-45. DOI: 10.1046/j.13652168.2002.02083.x

14. Westphalen A, Faria Araújo A, Zacharias P, et al. Repair of large incisional hernias. To drain or not to drain. Randomized clinical trial. Acta Cir Bras. 2015;30(12):844-51. DOI: 10.1590/S0102865020150120000009

15. Baroudi R, Alberto C, Ferreira A. Seroma: How to Avoid It and How to Treat It Seroma. Am Soc Aesthetic Plast Surg. 1998; (December):439-41. DOI: 10.1016/S1090-820X(98)70073-1

16. Martín-Duce A, Noguerales F, Villeta R, et al. Modifications to Rives technique for midline incisional hernia repair. Hernia. 2001;5(2):70-2. DOI: 10.1007/s100290100010

17.Vrijland W, Jeekel J, Steyerberg E, et al. Intraperitoneal polypropylene mesh repair of incisional hernia is not associated with enterocutaneous fistula. Br J Surg. 2000;87(3):348-52. DOI: 10.1046/j.1365-2168.2000.01364.x

18. Reiffel A, Pharmer L, Weinstein A, et al. A Prospective Analysis of the Association Between Indwelling Surgical Drains and Surgical Site Infection in Plastic Surgery. Ann Plast Surg. 2013;71(5):5615. DOI: 10.1097/SAP.0b013e31824c905b

19. Correa Alfonso R, Canals Rabaza P, Mederos Curbelo O, et al. Reparación de hernias incisionales grandes con malla supraaponeurótica. Rev Cuba Cir. 2002;41(3):156-63.

20. Fan J, Liu J, Chen K, et al. Preperitoneal closed - system suction drainage after totally extraperitoneal hernioplasty in the prevention of early seroma formation: a prospective double blind randomized controlled trial. Hernia. 2018;22(3):455-65. DOI: 10.1007/s10029-018-1731-2

$\mathrm{X}$

Tabla I. Características antropométricas y funcionales de los pacientes 


\begin{tabular}{|c|c|c|c|c|}
\hline & $\begin{array}{l}\text { Grupo A* } \\
\text { Con drenaje } \\
\text { (42) }\end{array}$ & $\begin{array}{l}\text { Grupo } B^{*} \\
\text { Sin drenaje } \\
(60)\end{array}$ & $\boldsymbol{p}$ & \\
\hline Sexo (F:M) & $32: 10$ & $33: 27$ & $\begin{array}{l}0.0 \\
28\end{array}$ & \\
\hline Edad (años) & $57 \pm 13$ & $53 \pm 12$ & $\begin{array}{l}0.1 \\
58\end{array}$ & $\begin{array}{l}\text { (n.s. } \\
\text { ) }\end{array}$ \\
\hline Peso (kg) & $86.4 \pm 20.1$ & $86.7 \pm 16.4$ & $\begin{array}{l}0.9 \\
35\end{array}$ & (n.s. \\
\hline Talla $(\mathrm{cm})$ & $165.5 \pm 7.9$ & $167.4 \pm 7.4$ & $\begin{array}{l}0.2 \\
20\end{array}$ & (n.s. \\
\hline IMC & $31.5 \pm 7.2$ & $30.9 \pm 5.7$ & $\begin{array}{l}0.6 \\
51\end{array}$ & $\begin{array}{l}\text { (n.s. } \\
)^{\prime}\end{array}$ \\
\hline Hemoglobina $(\mathrm{g} / \mathrm{dL})$ & $13.5 \pm 1.1$ & $13.7 \pm 1.5$ & $\begin{array}{l}0.5 \\
01\end{array}$ & (n.s. \\
\hline $\begin{array}{l}\text { Glóbulos blancos } \\
\left(/ \mathrm{mm}^{3}\right)\end{array}$ & $7503 \pm 2946$ & $7874 \pm 1939$ & $\begin{array}{l}0.4 \\
45\end{array}$ & (n.s. \\
\hline Urea (g/L) & $0.4 \pm 0.1$ & $0.4 \pm 0.2$ & $\begin{array}{l}0.4 \\
27\end{array}$ & (n.s. \\
\hline Creatinina $(\mathrm{mg} / \mathrm{dL})$ & $0.8 \pm 0.2$ & $1.1 \pm 0.9$ & $\begin{array}{l}0.2 \\
68\end{array}$ & $\begin{array}{l}\text { (n.s. } \\
)^{\prime}\end{array}$ \\
\hline
\end{tabular}

*Valores expresados en media \pm DS; n.s.: diferencia estadísticamente no significativa.

Se observó una diferencia estadísticamente significativa entre ambos grupos con respecto a la distribución por sexo ( $<<0.05$ ), con una mayor proporción de pacientes de sexo femenino en el grupo con drenaje.

Tabla II. Tamaño de las eventraciones 


\begin{tabular}{|l|l|l|l|}
\hline Tamaño & Con drenaje & Sin Drenaje & p \\
\hline Menor $3 \mathrm{~cm}$ & $0(0 \%)$ & $6(10 \%)$ & 0.035 \\
\hline $3-6 \mathrm{~cm}$ & $9(21.4 \%)$ & $31(51.6 \%)$ & 0.002 \\
\hline $6-10 \mathrm{~cm}$ & $14(33.3 \%)$ & $8(13.3 \%)$ & 0.016 \\
\hline$>10 \mathrm{~cm}$ & $19(45.2 \%)$ & $15(25 \%)$ & 0.033 \\
\hline Total general & $42(100 \%)$ & $60(100 \%)$ & \\
\hline
\end{tabular}

Los defectos más frecuentes encontrados fueron menores a $6 \mathrm{~cm}$. Encontramos diferencias estadísticamente significativas respecto a la colocación de drenaje y al tamaño de la eventración.

Tabla III. Cantidad de pacientes con complicaciones

\begin{tabular}{|l|l|l|}
\hline & Con drenaje & Sin drenaje \\
\hline Pacientes sin complicaciones & $21(50 \%)$ & $33(55 \%)$ \\
\hline Pacientes con complicaciones & $21(50 \%)$ & $27(45 \%)$ \\
\hline Total general de pacientes & $42(100 \%)$ & $60(100 \%)$ \\
\hline \multicolumn{2}{|l|}{ No encontramos diferencia estadísticamente } & significativa en el
\end{tabular}
desarrollo de complicaciones en función de la colocación de drenaje $(p=0.619)$. 
Tabla IV. Factores predisponentes

\begin{tabular}{|c|c|}
\hline $\begin{array}{l}\text { Factores de riesgo en la aparición de } \\
\text { complicaciones }\end{array}$ & $\mathbf{p}$ \\
\hline \multirow{2}{*}{ (1) } & 0.808 \\
\hline & (n.s.) \\
\hline Edad ( $\geq 50$ años) & 0.008 \\
\hline Diabetes & 0.003 \\
\hline \multirow{2}{*}{ Neoplasia } & 0.058 \\
\hline & (n.s.) \\
\hline Indice de masa corporal $(<30 / \geq 30)$ & 0.039 \\
\hline \multicolumn{2}{|l|}{ Tamaño de la eventración } \\
\hline \multirow{2}{*}{$3-6 \mathrm{~cm}$} & 0.120 \\
\hline & (n.s.) \\
\hline \multirow{2}{*}{$6-10 \mathrm{~cm}$} & 0.256 \\
\hline & (n.s.) \\
\hline$\geq 10 \mathrm{~cm}$ & 0.001 \\
\hline Drenaje ( Sí / No) & $\begin{array}{l}0.619 \\
\text { (n.s.) }\end{array}$ \\
\hline
\end{tabular}

La edad, la diabetes, el IMC y las eventraciones de mayor tamaño fueron los factores en los que se observó una asociación estadísticamente significativa con el desarrollo de complicaciones. 